\title{
ON RESIDUALLY FINITE GENERALIZED FREE PRODUCTS
}

\section{R. J. GREGORAC}

Let $S=A_{U}^{*} B$ be a nontrivial generalized free product of the groups $A$ and $B$ with amalgamated subgroup $U$ and suppose $A$ and $B$ are residually finite groups. Baumslag [1] has given conditions sufficient for $S=A_{U}^{*} B$ to itself be residually finite and these have been used to investigate the residual finiteness of $S$ by Baumslag [1] and Dyer [2], when the factors $A$ and $B$ of $S$ are assumed to satisfy certain additional properties. The question then arises as to the necessity of these conditions. It is shown here that Baumslag's conditions are in fact necessary, provided $A$ and $B$ satisfy suitable identical relations.

A group $G$ is residually finite if there exists a set $\left\{G_{i} \mid i \in I\right\}$ of normal subgroups of $G$ such that $G / G_{i}$ is finite for each $i \in I$ and $\bigcap_{i \in I} G_{i}$ $=1$; the set $\left\{G_{i} \mid i \in I\right\}$ is called a filter of $G[1]$.

Baumslag has shown

Theorem 1 [1]. Suppose $A$ and $B$ are residually finite groups with filters $\left\{A_{i} \mid i \in I\right\}$ and $\left\{B_{j} \mid j \in J\right\}$, respectively. The group $S=A_{U}^{*} B$ will be residually finite provided

(i) $\left\{A_{i} \cap U \mid i \in I\right\}=\left\{B_{j} \cap U \mid j \in J\right\}$ and

(ii) $\bigcap_{I} A_{i} U=U=\bigcap_{J} B_{j} U$.

Now suppose the nontrivial product $S=A_{U}^{*} B$ is residually finite with filter $\left\{S_{k} \mid k \in K\right\}$. Set $A_{k}=S_{k} \cap A$ and $B_{k}=S_{k} \cap B$ for each $k \in K$. Clearly $\left\{A_{k} \mid k \in K\right\}$ and $\left\{B_{k} \mid k \in K\right\}$ are filters of $A$ and $B$ satisfying (i) above. When do they also satisfy (ii)? Using the preceding notation we state

THEOREM 2. Let $w\left(x_{1}, \cdots, x_{n}\right)=1$ be a nontrivial identical relation on $B$. Then $\bigcap_{k \in K} A_{k} U=U$ if

(a) the index of $U$ in $B$ is greater than two, or

(b) $w\left(x_{1}, \cdots, x_{n}\right)=1$ is not an identical relation of the infinite dihedral group.

Proof. First note that $\cap S_{i}=1$ implies that any identical relation of $B$ is an identical relation of $\cap_{K} B S_{k}=R$, for the map defined by $r \mapsto\left(r S_{k}\right)$ is a monomorphism of $R$ in to the cartesian product $\prod B S_{k} / S_{k}$.

Received by the editors May 19, 1969. 
Now $R_{1}=\left\langle\bigcap_{K} U A_{k}, B>\subseteq R\right.$, because $A_{k} \subseteq S_{k}$, so $R_{1}$ satisfies the identical relation $w\left(x_{1}, \cdots, x_{n}\right)=1$. Put $X=\bigcap_{K} U A_{k}$. Then $R_{1}=X_{U}^{*} B$. That $X=U$ now follows from

Lemma 3. Suppose the generalized free product $G=H_{K}^{*} L$, where $H \neq K \neq L$, satisfies a nontrivial identical relation. Then $[H: K]$ $=[L: K]=2$, and the infinite dihedral group satisfies the identical relation.

Proof. Suppose $[H: K]>2$ and let $h_{1}$ and $h_{2}$ be elements in two different cosets of $K$ in $H$ but not in $K$. Choose $y \in L \backslash K$. Then $h_{1} y h_{1} y$ and $h_{2} y h_{2} y$ freely generate a free subgroup $F$ of $G$, so in this case $G$ satisfies no nontrivial identical relation.

Thus if $G$ does satisfy a nontrivial identical relation, then $[H: K]$ $=[L: K]=2$, so $K$ is normal in both $H$ and $L$. Thus $G / K$, which is the infinite dihedral group, must satisfy the identical relation as required.

As examples we note

THEOREM 4. Let $A$ and $B$ be finitely generated infinite nilpotent groups. Then $S=A_{U}^{*} B$ is residually finite if and only if

(i) $A$ and $B$ have normal series $A=A_{0} \supseteq A_{1} \supseteq \cdots, B=B_{0}$ $\supseteq B_{1} \supseteq \cdots$, such that $1<\left[A_{i}: A_{i+1}\right],\left[B_{i}: B_{i+1}\right]<\infty$ for all $i$,

(ii) $\cap_{i} A_{i}=1=\bigcap_{i} B_{i}$

(iii) $\left\{U \cap A_{i}\right\}=\left\{U \cap B_{i}\right\}$ and

(iv) $\bigcap_{i} U A_{i}=U=\bigcap_{i} U B_{i}$.

THEOREM 5. Let $A$ and $B$ be finitely generated infinite nilpotent groups and let $p$ be a prime. Then $S=A_{U}^{*} B$ is residually a finite p-group if and only if (i) $A$ and $B$ have normal series $A=A_{0} \supseteq A_{1} \supseteq \cdots, B=B_{0}$ $\supseteq B_{1} \supseteq \cdots$, such that $\left[A_{i}: A_{i+1}\right]=p=\left[B_{i}: B_{i+1}\right]$ for all $i$, and such that (ii), (iii) and (iv) of Theorem 4 hold.

Corollary 6. Let $S=g p\left(a, b \mid a^{h}=b^{k}\right)$ and let $p$ be a prime. Then $S$ is residually a finite p-group if and only if

(a) both $h$ and $k$ are powers of $p$, or

(b) $h=1$ or $k=1$.

Theorem 4 follows immediately from Theorem 1 and Theorem 2 . Theorem 5 follows from an easy extension of Theorem 1 using the main result of Higman [3]. The (well-known) corollary to Theorem 5 follows because property (iv) fails when $h \neq 1$ and $k \neq 1$ are not both powers of $p$.

Although special cases of Lemma 3 are well known (see for example $[4$, p. 217, Problem 10]), the proof of the general case given here is 
due to Professor B. H. Neumann. I thank Professor Neumann for his help with this.

\section{REFERENCES}

1. G. Baumslag, On the residual finiteness of generalised free products of nilpotent groups. Trans. Amer. Math. Soc. 106 (1963), 193-209. MR 26 \#2489.

2. J. L. Dyer, On the residual finiteness of generalized free products, Trans. Amer. Math Soc. 133 (1968), 131-143.

3. G. Higman, Amalgams of p-groups, J. Algebra 1 (1964), 301-305. MR 29 \#4799.

4. W. Magnus, A. Karrass and D. Solitar, Combinatorial group theory: Presentations of groups in terms of generators and relations, Pure and Appl. Math., vol. 13, Interscience, New York, 1966. MR 34 \#7617.

The Australian National University, Canberra 